\title{
The Ocean Wave Directional SPECTRUM
}

\author{
By Lucy R. Wyatt
}

$\mathrm{T}$ he directional SPectrum $\mathrm{S}(\mathrm{k})$ [or $\mathrm{S}(\mathrm{f}, \theta)]$ measures the distribution of wave energy in wave number, $k$, (or frequency, $f$ ) and direction. Different contributions to local wave energy, e.g., swell from distant storms and locally wind-generated waves, can be identified in a measurement of the directional spectrum (see Fig. 1). The direction of propagation of wave energy and the period (1/f) of the most energetic waves are important for many practical applications, e.g., the design and operation of coastal and offshore structures and storm surge forecasts.

The use of $\mathrm{HF}$ radar to make measurements of the spectrum is based on equations developed by Barrick $(1972 \mathrm{a}, \mathrm{b})$ that relate the power spectrum of the backscattered signal to the ocean wave spectrum through a nonlinear integral equation. The backscattered signal is dominated by Bragg scattering, i.e., the ocean waves responsible for the scatter have a wavelength of one-half the radio wavelength. The measurement of the directional spectrum is only possible because ocean waves are not simple sinusoidal forms satisfying the linear dispersion relationship. If they were linear there would be only the two first-order peaks in the backscattered power (Doppler) spectrum. The nonlinear properties of ocean waves give rise to waves with the correct wavelength for Bragg scatter but with different frequencies and hence different Doppler shifts. This is the second-order part of the spectrum described by Barrick's integral equation.

To make the measurement, the integral equation must be solved. This is not straightforward and a number of methods have been proposed (Lipa, 1978; Wyatt, 1990; Howell and Walsh, 1993; Hisaki, 1996). No method as yet has gained widespread acceptance and there are no operational $\mathrm{HF}$ radars making routine wave measurements. Here we concentrate on the Wyatt method and show some measurements from the Ocean Surface Current Radar (OSCR) HF radar system

Lucy R. Wyatt. Sheffield Centre for Earth Observation Science School of Mathematics and Statistics (AM). University of Sheffield, Hounsfield Road. Sheffield S3 7RH. UK. that is marketed by Marconi Radar Co. as a surface current measurement radar and has a maximum range of $\sim 20 \mathrm{~km}$ for wave measurement. The method has also been used with two different radar systems: Pisces, a system developed by Neptune Radar Ltd. from a University of Birmingham, U.K., prototype that has a maximum range of $\sim 150 \mathrm{~km}$ for wave measurement (Wyatt, 1991), and WERA (WEllen RAdar), a system under development at the Institut für Meereskunde. University of Hamburg, Germany, with a maximum range similar to OSCR.

\section{The Method}

The concept of the method is simple. Significant waveheight and wind direction are estimated from the radar data using methods referred to elsewhere in this issue (Fernandez et al.. 1997; Graber and Heron. 1997; see also Wyatt 1988; Wyatt et al., 1997). These are used to derive a model directional spectrum using both the waveheight to determine a Pierson-Moskowitz wave number spectrum and also the wind direction in a directional distribution. This model spectrum is fed into the integral equation that is integrated directly to determine the corresponding Doppler spectrum. Differences between this Doppler spectrum and the measured spectrum are used to modify the directional spectrum at each wave number (within a limited range) and direction. This process is repeated until the differences become sufficiently small. The way in which the modification is carried out is the key to the success of the method. The details are too complicated to be included here, but it is important to realize that although the method starts with a simple directional spectrum with a single wind-wave mode, the solution (when the procedure has converged) can be very different; for example, it has no problem detecting the presence of swell propagating in a very different direction.

The method does not provide a measurement of the directional spectrum at all wave frequencies. High-frequency waves are not adjusted in the procedure and retain the memory of the initializing spectrum. The cutoff, at OSCR frequencies, is

\section{The measurement}

of the directional

spectrum is only

possible because

ocean waves are not simple sinusoidal

forms . . . 


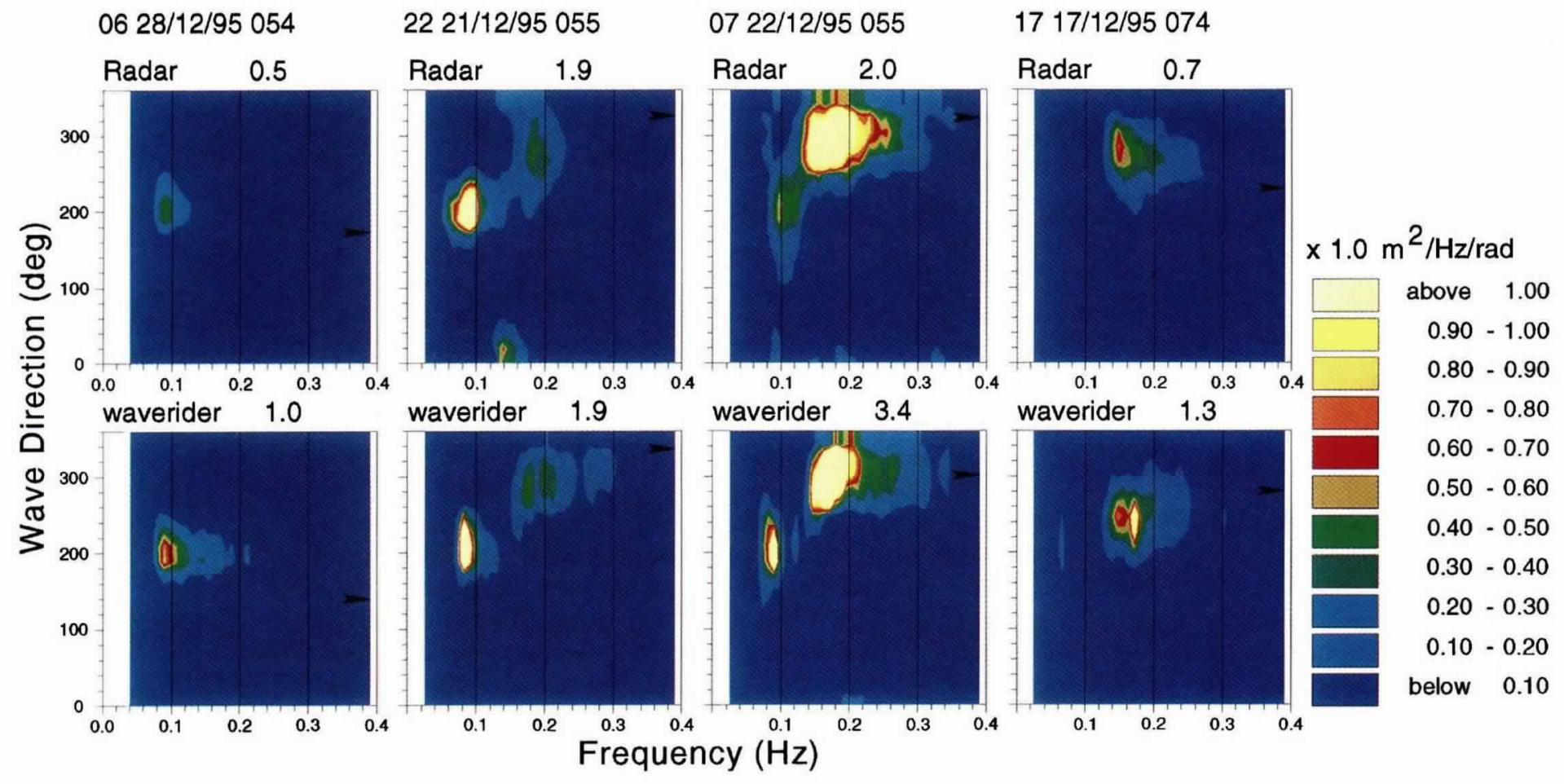

Fig. 1: Directional spectra measured by the radar (top row) and estimated from the directional waverider measurement (bottom row) with frequency in $\mathrm{Hz}$ on the horizontal axis and direction toward which the waves are propagating on the vertical. Four different types of wavefield are shown: (left) swell-dominated sea with a peak frequency of $0.09 \mathrm{~Hz}$; (mid-left) swell $(0.09 \mathrm{~Hz})$ with some wind sea with a peak frequency $\sim 0.18 \mathrm{~Hz}$; (mid-right) wind-sea $(0.16 \mathrm{~Hz})$ with some swell $(0.09 \mathrm{~Hz})$; (right) wind-sea peaking at $0.14 \mathrm{~Hz}$. The hour and date of each measurement is shown together with the cell location of the radar measurement (all close to the wavebuoy site). The number above each plot is the peak in the directional spectrum for that plot. Arrow heads, short wave direction. The color coding is indicated on the right: dark blue, $<0.1 ;$ yellow, $>1.0$.

\section{One advantage of}

this method is that

wave components in

the spectrum that are

similar in frequency

but different in

direction emerge

naturally during the

inversion. $\sim 0.38 \mathrm{~Hz}$, the exact value depending on the look directions of the two radars at the measurement point. The Lipa, Howell, and Walsh methods have similar limitations, the exact cutoff in each case depends on the model assumed for high frequencies and on the range of Doppler frequencies included in their analyses. Hisaki's method is a nonlinear optimization method and in principle extends the range of frequencies, although the method has not been exhaustively verified. For most applications it is the longer, lower-frequency, energy-containing waves that are of interest. Wind speeds would have to be very low or fetch very short for the peak in the spectrum to be at frequencies close to the cutoff.

An important difference between the Wyatt method and those of Lipa, Howell and Walsh, and Hisaki is the form of the solution. In the other three methods the directional spectrum is discretized in scalar wave number or frequency and is expressed as a truncated Fourier series in direction, which allows the problem to be expressed as a matrix equation for the Fourier coefficients. The Fourier coefficients used are the same five used in the analysis of directional wavebuoys; methods employed in the interpretation of buoy data can be applied directly. One limitation is difficulty in identifying wave components in the spectrum that are similar in frequency but different in direction. This, of course, applies both to the wavebuoy and the radar analysis. In the Wyatt method the directional spectrum is discretized in vector wave number and the solution is determined at each discrete vector wave number. One advantage of this method is that wave components in the spectrum that are similar in frequency but different in direction emerge naturally during the inversion (Wyatt and Holden, 1994). Validating the detailed structure in the radar-measured spectrum is not straightforward because wavebuoys only measure limited detail. One approach is to partition the wave spectra into swell and windwave modes and compare integrated parameters for these. Such an approach is under development (Isaac and Wyatt, 1997).

\section{Holderness Measurements}

The data set used here to demonstrate the success of the method was collected using the OSCR HF radar during a deployment on the U.K. coast at Holderness. Directional waveriders were deployed at two offshore locations about midway between the radar sites, one in $\sim 10$ and the other in $\sim 20 \mathrm{~m}$ water depth. Depth contours are roughly parallel to the coast deepening to $10 \mathrm{~m} \sim 1 \mathrm{~km}$ offshore. The 


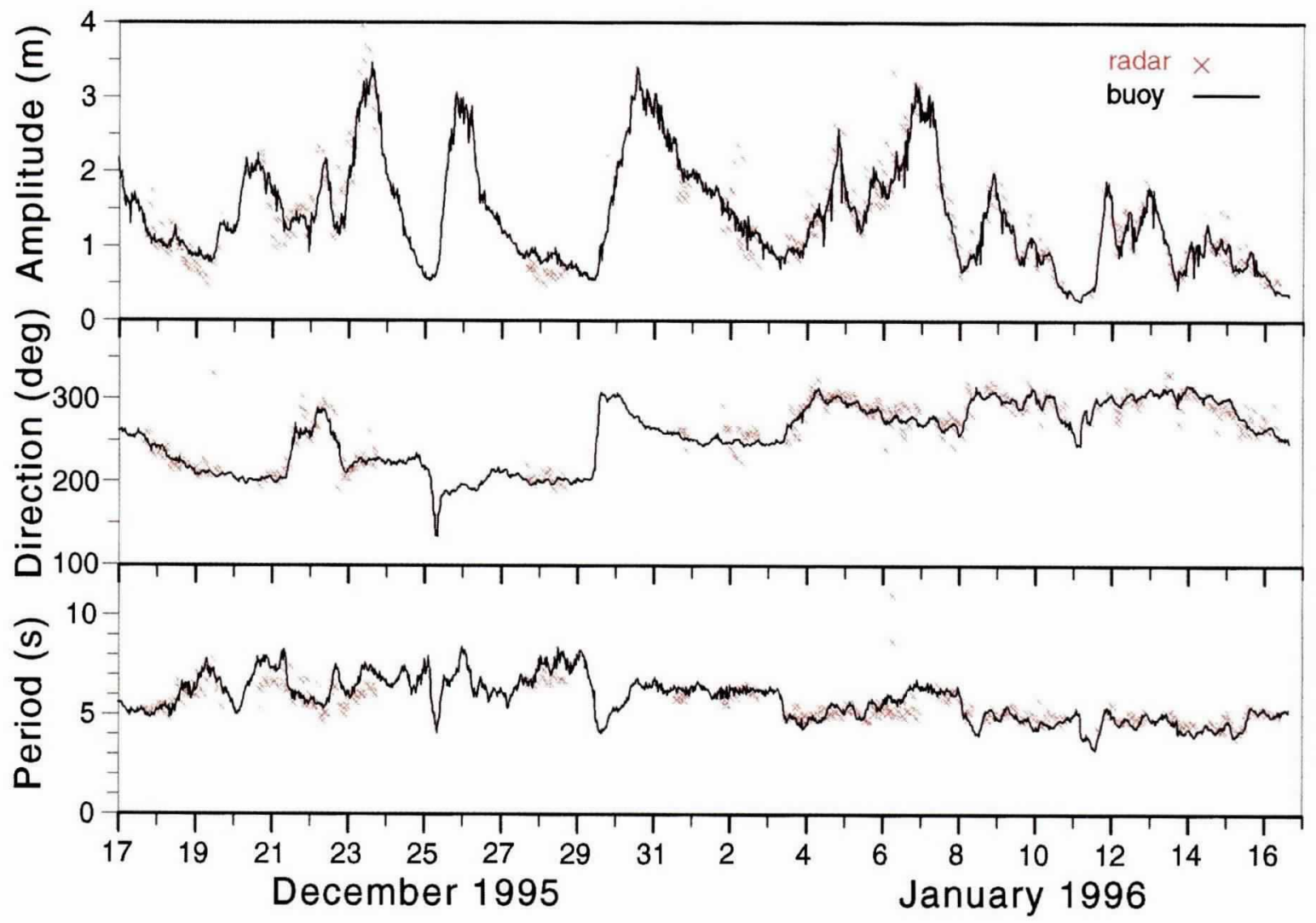

Fig. 2: Time series of amplitude (significant waveheight), mean direction, and mean period obtained from the directional spectra measured by the radar $(X)$ and from the directional parameters measured by the waverider (-) in both cases integrated over the frequency band of $0.05-0.4 \mathrm{~Hz}$.

OSCR system used was provided by Wimpey Environmental (now GEOS Ltd.). Data were collected over a period of 1 mo from 17 December 1995 to 16 January 1996 with short breaks over the Christmas-New Year period (the data requirements for wave measurement require an operator to be on site). The radar measurement period included two high sea-state events, high for this region at least, with significant waveheights reaching $>3 \mathrm{~m}$ at the buoy positions (Fig. 1). Surprisingly for this region, there were very few examples of fetch-limited development. Figure 2 shows time series comparisons of significant waveheight, mean direction, and mean period obtained from the directional spectrum measured by the radar and a co-located wavebuoy; very good agreement can be seen. Similar comparisons can be made for these parameters measured over limited frequency bands; for example, waves with frequencies $<0.1$ $\mathrm{Hz}$, which, during this experiment at least, are swell waves. Again good agreement is found. Quantitative measures of the accuracy of a range of parameters describing the directional spectrum are being determined.

One big advantage HF radar has over conventional wave measurement systems is the ability to monitor wave development in space as well as over time. This advantage is demonstrated very clearly with data collected on 21 December 1995 in the early afternoon, a relatively calm period with high pressure over eastern Europe, low pressure over Scandinavia and to the west of the British Isles, driving a weak south-easterly wind

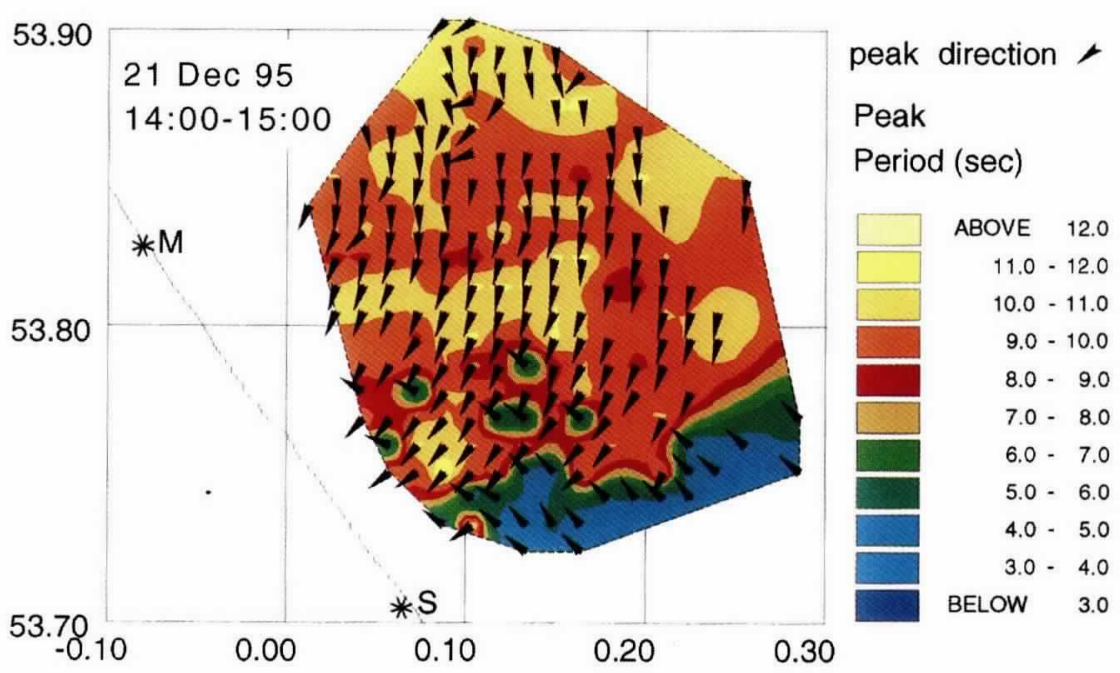

Fig. 3: The Holderness coastline is shown to the south-west of the map with the two radar sites indicated as $M$ and $S$. Directions shown with arrowheads are those toward which the peak of the radar measured spectrum is propagating. The period of the peak is color coded as shown. This figure shows swell dominating over most of the region propagating from the north and refracting toward the coast. To the south, south-easterly wind waves dominate. 


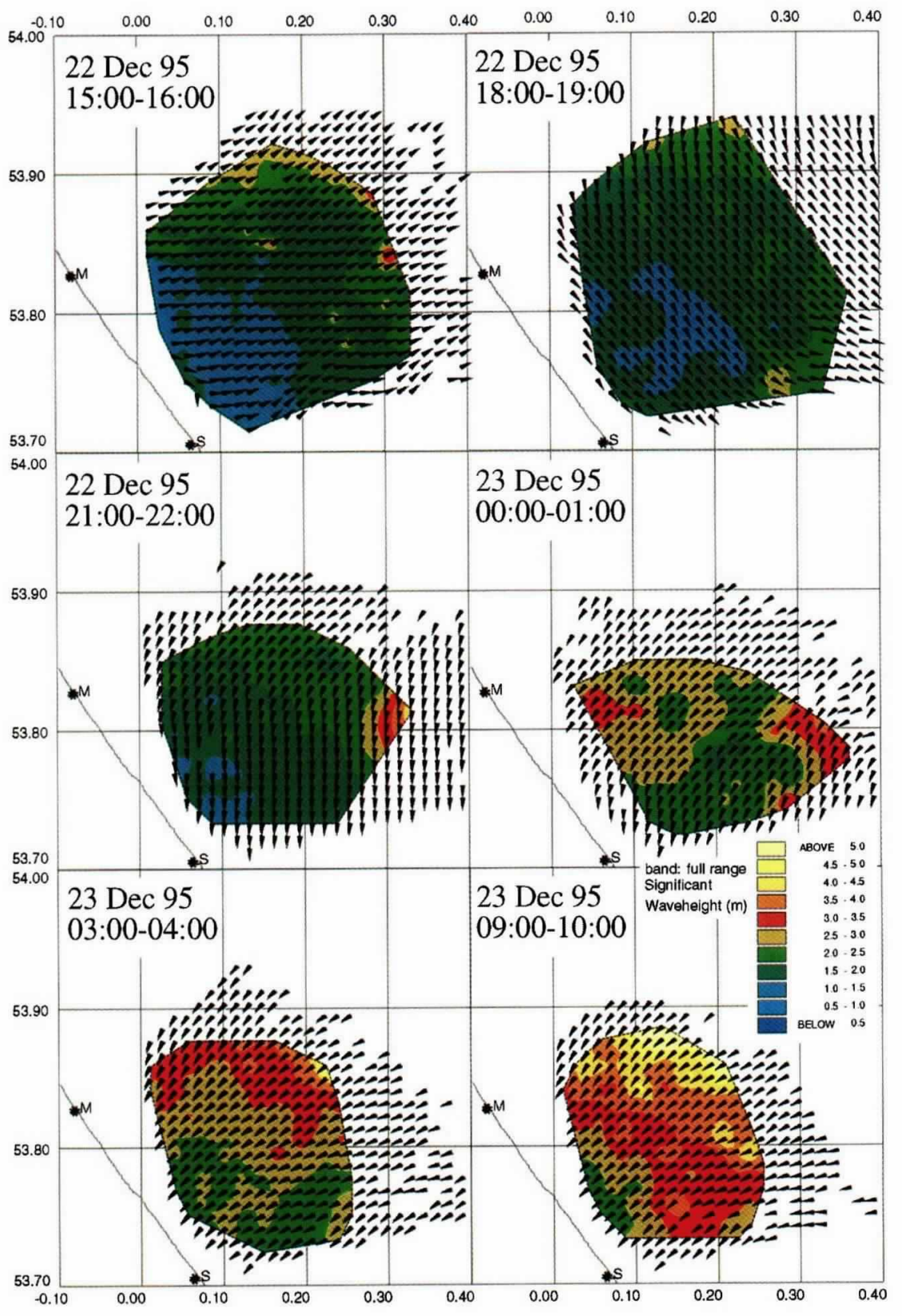

Fig. 4: Short-wave (wind) directions are shown with arrowheads. Significant waveheight is color-coded as shown and varies from $<1.0 \mathrm{~m}$ near to the shore on 22 December 1995 at 1500 to $>5$ m offshore at 0900 on $23 \mathrm{De}$ cember 1995. The figure shows changes because of the passage of a lowpressure system through the region.

\section{The data presented}

here have demon-

strated that OSCR

can measure the

ocean wave direc-

tional spectrum. over the region. Fronts were building up in the west to come through the region over the next 24 $\mathrm{h}$, but in the meantime the synoptic pattern suggested that the wave field would be dominated by swell propagating southward down the North Sea. This pattern is exactly the same as seen over most of the radar coverage region. Figure 3 shows the direction of the peak of the spectrum superimposed on the contoured peak period. Of interest is the spatial variation in these parameters. Swell, with a period of $\sim 10 \mathrm{~s}$ (yellow shades), can be seen propagating from the north and being refracted toward the coast as bottom depth de- creases. Swell energy is dissipated (not shown here), probably by bottom friction or by wave-current interaction. Tidal current speeds were large at the time of the measurement $(\geq 0.5 \mathrm{~m} / \mathrm{s})$, and although uniform in direction themselves (in roughly a southward direction), the direction relative to the swell varies as the swell is refracted by the bottom topography. Wave breaking is unlikely to be a problem at these amplitudes in these depths. As a result of the dissipation of swell energy, the spectral maximum in the south of the region shifts to the wind-wave field with a peak period of $\leq 5 \mathrm{~s}$ (blue shades on the figure). Comparisons of amplitude, direction, and spread as functions of frequency measured by the radar and wave buoy confirm the swell and wind-sea directions and also show good agreement in relative amplitudes at the buoy location.

A second example showing both temporal and spatial variability is presented in Figure 4 and shows significant waveheight determined from the directional spectra and short-wave directions (Wyatt et al., 1997) at each measurement position. Short-wave directions can be made over a wider area because they use only the first order part of the Doppler spectrum. The figure shows the response of the wave field to a passing low-pressure system. Waveheights are initially low in response to offshore winds but increase after the low has passed through, leaving a strong north-easterly wind pattern and hence longer fetch.

\section{Concluding Remarks}

The data presented here have demonstrated that OSCR can measure the ocean wave directional spectrum. These OSCR measurements are much better than those previously published (Wyatt and Ledgard, 1996) because this Holderness deployment configuration was optimized for wave measurement. Good agreement with wave buoy parameters was shown and the comparison is now the subject of rigorous evaluation to provide quantitative measures of parameter accuracy. Oceanographic consistency of the data over the region of the measurements is also being demonstrated.

There remain some problems that need to be overcome before OSCR can become an operational tool for wave monitoring. Some of these are purely technical; for example, the use of modern computer systems and, in particular, reliable and high-capacity data storage devices would significantly increase the temporal coverage because there would no longer be an onsite operator requirement. Others require further research, some of which is currently underway at Sheffield. One of the problems we have identified is a reduction in data availability during periods of varying surface current. This is because the first order peaks move about in frequency during the measurement period (responding to different current components) and prevent a clear distinction between first 
and second order in the power spectrum. At present OSCR data from three separate surface current measurement periods (10 min in every 20 ) are averaged before extracting wave information (a statistical requirement). Any current variability on time scales of $<1 \mathrm{~h}$ causes a problem. This could be overcome by longer coherent data collections. thus obtaining sufficient averaging for wave measurement from a shorter time period, but at the expense of reduced surface current sampling. Other approaches to this problem are under development. Another important problem, identified many years ago by Lipa and Barrick (1986), is a possible upper waveheight limit beyond which the theory used in the inversion no longer applies. So far we have seen no evidence of the particular problem they describe, although there are certainly differences between the second-order theory and the radar measurements in higher sea-states that may be affecting the accuracy of our measurements (Wyatt, 1995). Measurements are required in seastates higher than are seen in the Holderness region to investigate these issues further. Recent measurements with the WERA system off the north Netherlands' coast at Petten during a very stormy period in November 1996 may provide some answers.

\section{Acknowledgments}

The observations off Holderness described here were funded in part by the Ministry of Agriculture Fisheries and Food (U.K.) under its Flood Protection Commission with NERC's Proudman Oceanographic Laboratory. The data were collected and analyzed by Louise Ledgard. The Natural Environment Research Council also supported these observations through its Land Ocean Interaction Studies project. Other support has come from the EC MAST SCAWVEX project (MAS2CT940103) and EPSRC (grants GR/J07341, GR/J50934).
References

Barrick, D.E., 1972a: First-order theory and analysis of MF/HF/VHF scatter from the sea. IEEE Trans. Antennas Propag., AP-20, 2-10.

1972b: Remote sensing of sea state by radar. In: $R e-$ mote Sensing of the Troposphere. V.E. Derr. ed. NOAA/Environmental Research Laboratories, Boulder. CO. 12.1-12.6.

Fernandez. D.M., H.C. Graber. J.D. Paduan and D.E. Barrick. 1997: Mapping wind direction with HF radar. Oceanography, 10. 93-95.

Graber, H.C. and M.L. Heron, 1997: Waveheight measurements from HF radar. Oceanography, 10, 90-92

Hisaki. Y.. 1996: Nonlinear inversion of the integral equation to estimate ocean wave spectra form $\mathrm{HF}$ radar. Radio Sci. 31, 25-39.

Howell, R. and J. Walsh. 1993: Measurement of ocean wave spectra using narrow beam HF radar. IEEE J. Ocean. Eng.. 18, 296-305.

Isaac, F.E. and L.R. Wyatt, 1997: Segmentation of HF radar measured directional wave spectra using the Voronoi Diagram. J. Atmos. Ocean. Tech., 14, 950-959.

Lipa, B.J., 1978: Inversion of second-order radar echoes from the sea. J. Geophys. Res., 83, 959-962.

and D.E. Barrick, 1986: Extraction of sea state from HF radar sea echo: mathematical theory and modelling. Radio Sci., 21, 81-100.

Wyatt, L.R.. 1988: Significant waveheight measurement with HF radar. Int. J. Remote Sens., 9, 1087-1095.

- 1990: A relaxation method for integral inversion applied to HF radar measurement of the ocean wave directional spectrum. Int. J. Remote Sens,, II, 14811494.

1991: HF radar measurements of the ocean wave directional spectrum. IEEE J. Ocean. Eng., I6, 163-169. 1995: High order nonlinearities in HF radar backscatter from the ocean surface. In: IEE Proceedings on Radar, Sonar and Navigation, 142, IEE, London. 293-300.

- and G.J. Holden, 1994: HF radar measurement of multi-modal directional wave spectra. Global Atmos. Ocean Sys., 2, 265-290.

and L.J. Ledgard, 1996: OSCR wave measurementsome preliminary results. IEEE J. Ocean. Eng., 21, 6476.

. L.J. Ledgard and C.W. Anderson, 1997: Maximum likelihood estimation of the directional distribution of $0.53 \mathrm{~Hz}$ ocean waves. J. Atmos. Ocean. Tech. 14, 591-603. 